\title{
Atendimentos odontológicos durante a pandemia da COVID-19 e as medidas de biossegurança adotadas: revisão integrativa
}

\author{
Dental care during the COVID-19 pandemic and adopted biosafety measures: integrative review \\ Atención odontológica durante la pandemia de COVID-19 y medidas de bioseguridad adoptadas: \\ revisión integradora
}

Recebido: 21/01/2021 | Revisado: 27/01/2021 | Aceito: 01/02/2021 | Publicado: 09/02/2021

Bruna de Carvalho da Costa Pereira

ORCID: https://orcid.org/0000-0003-4852-4519

Universidade de Pernambuco, Brasil

E-mail: brunaaccp@gmail.com

Maria Luiza Dornelas de Albuquerque Aragão

ORCID: https://orcid.org/0000-0002-3739-0669

Universidade de Pernambuco, Brasil

E-mail: luizadaragao@hotmail.com

Renata Araújo Gomes de Sá

ORCID: https://orcid.org/0000-0001-6550-8225

Universidade de Pernambuco, Brasil

E-mail: renata.sa@upe.br

Eloiza Leonardo de Melo

ORCID: https://orcid.org/0000-0001-5845-1478

Universidade de Pernambuco, Brasil

E-mail: eloiza.leonardo@upe.br

Marleny Elizabeth Márquez de Martínez Gerbi ORCID: https://orcid.org/0000-0001-9174-2541

Universidade de Pernambuco, Brasil E-mail: marleny.gerbi@upe.br

Esdras Gabriel Alves-Silva

ORCID: https://orcid.org/0000-0002-2309-1115

Universidade Estadual de Campinas, Brasil E-mail: esdras0702@yahoo.com.br

Mávio Eduardo Azevedo Bispo ORCID: https://orcid.org/0000-0002-3781-0272 Universidade de Pernambuco, Brasil E-mail: meb@ecomp.upe.br

Maria Regina Almeida de Menezes ORCID: https://orcid.org/0000-0003-3012-3979 Universidade de Pernambuco, Brasil E-mail: regina.menezes@upe.br

\begin{abstract}
Resumo
Com a pandemia da COVID-19, causada pelo novo coronavírus, adaptações foram feitas no mundo para que possamos conviver com esse vírus, enquanto cientistas procuram uma forma eficaz de combatê-lo. Na odontologia, cirurgiõesdentistas tiveram que se reinventar nos seus atendimentos para que eles fossem realizados de forma segura, acrescentando novas medidas de biossegurança. $\mathrm{O}$ objetivo do presente trabalho foi realizar uma revisão integrativa da literatura atual, a fim de entender o efeito do coronavírus quanto à introdução das novas medidas de biossegurança para os atendimentos odontológicos, considerando os benefícios, limitações e impactos para a sociedade. Trata-se de uma revisão integrativa de literatura baseada em uma pesquisa bibliográfica de artigos, nas bases de dados PubMed, LILACS, BVS, Scopus e Cochrane Library. Após os critérios de elegibilidade, foram incluídos 15 artigos, sendo 11 publicados em língua inglesa, 2 em língua portuguesa e 2 em língua espanhola. Posterior a análise dos artigos, suas respectivas sínteses foram apresentadas em um quadro. Com isso, fica evidente que os benefícios do uso dos equipamentos e estratégias de biossegurança, principalmente os equipamentos de proteção individual (EPI) e o isolamento social, são primordiais para o bem-estar. Ademais, o tema abordado neste trabalho se torna indispensável, visto que ações de biossegurança sempre foram almejadas nos atendimentos, a fim de diminuir a contaminação infectocontagiosa, sobretudo no momento atual.
\end{abstract}

Palavras-chave: Contenção de riscos biológicos; Odontologia; Infecções por coronavirus; Coronavirus; Controle de infecções. 


\begin{abstract}
Due to the COVID-19 pandemic, caused by the new coronavirus, adaptations have been made around the world so that it would be possible to live with this virus while scientists are looking for an effective way to combat it. In dentistry, dental surgeons had to reinvent themselves in their procedures in order to be carried out safely, adding new biosafety measures. The objective of the present survey was to carry out an integrative review of the current literature in order to understand the effect of the coronavirus regarding the introduction of new biosafety measures for dental care, considering the benefits, limitations and impacts for society. It is an integrative literature review that was based on a bibliographic search of articles, in the databases PubMed, LILACS, BVS, Scopus and Cochrane Library. After the eligibility criteria, 15 articles were included, 11 published in English, 2 in Portuguese and 2 in Spanish. After analyzing the articles, their respective syntheses were presented on a board. Herewith, it is concluded that the benefits of using biosafety equipment and strategies, especially personal protective equipment (PPE) and social isolation are paramount to well-being. In addition, the theme addressed in this work becomes indispensable, since biosafety actions have always been sought in order to reduce infectious contagious contamination and especially at the present time.
\end{abstract}

Keywords: Containment of biological risks; Dentistry; Coronavirus infections; Coronavirus; Infection control.

\title{
Resumen
}

Con la pandemia del COVID-19, causada por el nuevo coronavirus, fueron hechas adaptaciones en el mundo para que podamos convivir con este virus, mientras los cientistas buscan una forma eficaz de combatirlo. En la odontología, dentistas cirujanos tuvieron que reinventarse en sus asistencias para que estas fueran realizadas de manera segura, adicionando nuevas medidas de bioseguridad. El objetivo del trabajo presente fue realizar una revisión integral de la literatura actual, con el objetivo de entender el efecto del coronavirus en relación a la introducción de nuevas medidas de bioseguridad para las asistencias odontológicas, considerando los beneficios, limitaciones e Impacto a la sociedad. Se trata de una revisión integral de literatura que fue basada en una investigación bibliográfica de artículos, en las bases de datos PubMed, LILACS, BVS, Scopus y Cochrane Library. Después de los criterios de elegibilidad, fueron incluído 15 artículos, de los cuales 11 fueron publicados en lengua inglesa, 2 en lengua portuguesa, y 2 en lengua española. Posteriormente al análisis de los artículos, sus respectivas síntesis fueron presentadas en un cuadro. Con esto, queda evidente que los beneficios del uso de los equipamientos y estrategias bioseguridad, principalmente los equipamientos de protección individual (EPI) y el aislamiento social son primordiales para el bienestar. Además, el tema abordado en este trabajo se vuelve indispensable, dado que las acciones de bioseguridad siempre fueron deseadas en las asistencias, con fin de disminuir la contaminación infecto contagiosa, sobre todo en el momento actual.

Palabras clave: Contención de riesgos biológicos; Odontología; Infecciones por coronavirus; Coronavírus; Control de infecciones.

\section{Introdução}

A pandemia da COVID-19, causada pelo novo coronavírus (SARS-COV-2), teve seu princípio em Wuhan, na China. $\mathrm{O}$ aumento desenfreado de casos, globalmente, trouxe muitas consequências para a população, para tentar minimizar a disseminação do vírus as medidas comumente utilizadas foram o isolamento social, o uso de máscaras e lavar as mãos ou utilizar álcool em gel 70\% - 90\%. Essas estratégias foram muito efetivas em alguns países, por isso, várias mortes foram evitadas e a transmissão do vírus controlada (Guiñez-Coelho, 2020).

À medida que os cientistas foram obtendo informações concretas sobre o SARS-COV-2, foi possível traçar métodos de prevenção que fossem eficazes para a segurança da população. As ações de biossegurança são imprescindíveis nesse cenário pandêmico como uma forma de garantir o funcionamento seguro de locais que atendam ao público, prevenindo, controlando e até mesmo eliminando os riscos inerentes às atividades que possam interferir na qualidade de vida (Santos \& Barbosa, 2020).

O consultório odontológico, por se caracterizar como um serviço de saúde, é um local vulnerável a diversos microrganismos, existindo o risco de infecções cruzadas. Por isso, vários métodos já são adotados normalmente, mesmo antes da existência da pandemia do coronavírus, dentre eles o uso da máscara cirúrgica, jaleco, touca e óculos de proteção (Jurema et al., 2020; Kiliçarslan et al., 2020).

Com a existência da pandemia, as consultas odontológicas foram restritas aos atendimentos de urgência e, posteriormente, no começo do segundo semestre foram retomados os atendimentos eletivos, de acordo com a situação de cada local. Com isso, o medo, causado pela falta de informação sobre a transmissão desse vírus, proporcionou o surgimento de novas estratégias como aferição de temperatura antes da consulta, paciente fazer bochecho com peróxido de hidrogênio a $1 \%$ ou 1,5\% 
ou povidona a $0,2 \%$. Além de um rigor maior sobre a utilização das que já existem, desinfecção minuciosa das superfícies após cada atendimento e utilização completa dos equipamentos de proteção: gorro, óculos, máscara do tipo N95, face-shield, luvas e jaleco (Pereira et al., 2020).

Tendo em vista tal problemática, o objetivo dessa pesquisa foi verificar quais são as medidas de biossegurança adotadas, seus benefícios e limitações durante e após a pandemia do coronavírus para o atendimento odontológico, revelando o impacto para os serviços odontológicos públicos e privados, através de uma revisão integrativa. Espera-se que os conhecimentos adquiridos possam despertar o interesse da comunidade acadêmica e dos cirurgiões-dentistas clínicos em geral, de forma que as informações contribuam para mudança de atitudes de maneira mais consciente.

\section{Metodologia}

Trata-se de uma revisão integrativa da literatura, desenvolvida no ano de 2020, seguindo os seguintes passos: identificação do problema, busca na literatura (identificação das palavras-chaves, critérios de inclusão e exclusão, busca nas bases de dados), avaliação qualitativa, análise dos dados e apresentação dos resultados (Whittemore \& Knafl, 2005).

A indagação que norteou o estudo foi: quais são as medidas de biossegurança que estão sendo adotadas nos atendimentos odontológicos, durante a pandemia da COVID-19?

Os artigos foram coletados por meio das bases de dados PubMed/Medical Literature Analysis and Retrieval System Online (MEDLINE), Literatura Latino-americana e do Caribe em Ciências da Saúde (LILACS), Biblioteca Virtual de Saúde (BVS), Scopus e Cochrane Library. Os termos utilizados como palavras-chaves para a pesquisa foram: "Contenção de Riscos Biológicos", "Odontologia", "Infecções por Coronavirus", "Coronavirus" e "Controle de Infecções".

Para a base de dados LILACS foram utilizadas as estratégias de busca "Contenção de Riscos Biológicos AND Odontologia AND Infecções por Coronavirus" (estratégia A) e "Controle de Infecções AND Infecções por Coronavirus" (estratégia B). Para a base de dados BVS foram utilizadas novamente a estratégia de busca A e "Contenção de Riscos Biológicos AND Infecções por Coronavirus" (estratégia C). Para as bases de dados PubMed/MEDLINE, Scopus e Cochrane Library foi utilizada a estratégia de busca "Coronavirus AND Odontologia" (estratégia D).

\subsection{Critérios de elegibilidade dos artigos}

Foram incluídos artigos que estavam completos e disponíveis para download; com lapso temporal do último ano (2020) e relacionados ao tema desta revisão integrativa. Artigos repetidos, não disponíveis e em idiomas diferentes de inglês, português e espanhol foram excluídos.

\subsection{Seleção das publicações e extração dos dados}

Dois pesquisadores: B. C. C. P. e M. L. D. A. A., realizaram a seleção simultaneamente e de maneira independente. A avaliação inicial foi feita através da leitura do título e do resumo, e selecionados com base nos critérios de elegibilidade. Após isto, os artigos incluídos foram lidos na integra e as informações de interesse foram tabeladas.

A priori, foi organizado o compilado com os resultados em uma pasta na plataforma Google Drive, a fim de se conseguir o acesso de forma bilateral, com posterior comparação dos textos para obtenção daqueles selecionados por consenso. Artigos que apareciam repetidamente nas cinco bases de dados foram considerados apenas uma vez.

A fim de estimar a compatibilidade entre os dois pesquisadores no que se refere a concordância dos artigos finais, selecionados como base para produção da presente revisão integrativa, foi utilizado o teste de concordância Kappa (K). Tendo como princípio o conjunto de 20 artigos selecionados após a leitura superficial. 15 projetos foram aprovados pelos pesquisadores e 3 foram rejeitados, 1 projeto foi rejeitado só pelo pesquisador M. L. D. A. A. e 1 projeto foi rejeitado só pelo pesquisador B. 
C. C. P, totalizando os 20 artigos. O resultado da taxa de aceitação relativa foi 0,9 e a taxa hipotética de aceitação foi 0,027 . Realizando o cálculo do coeficiente K o resultado obtido foi 0,89 , estimado pela classificação de níveis de concordância de Landis e Koch (1977) como concordância perfeita $(0,81-1,0)$.

\section{Resultados}

Após a aplicação das estratégias de busca, um total de 161 artigos científicos foram encontrados. Após a leitura do título e resumo foram incluídos 20 artigos, e após a leitura na integra e aplicação dos critérios de inclusão e exclusão 15 artigos foram incluídos nesta revisão.

Na base de dados LILACS um total de 6 artigos foram encontrados, utilizando a estratégia B e 2 artigos utilizando estratégia A. Na base de dados BVS, 62 artigos foram encontrados utilizando a estratégia de busca C e 5 artigos encontrados utilizando a estratégia A. Nas bases de dados PubMed, Scopus e Cochrane Library foram encontrados 12, 73 e 1 artigos respectivamente, utilizando a estratégia C. Os detalhes encontram-se no Fluxograma 1. 
Research, Society and Development, v. 10, n. 2, e16010212248, 2021

(CC BY 4.0) | ISSN 2525-3409 | DOI: http://dx.doi.org/10.33448/rsd-v10i2.12248

Fluxograma 1: Detalhes da seleção dos artigos utilizando o método PRISMA.

\section{PRISMA 2009 Flow Diagram}
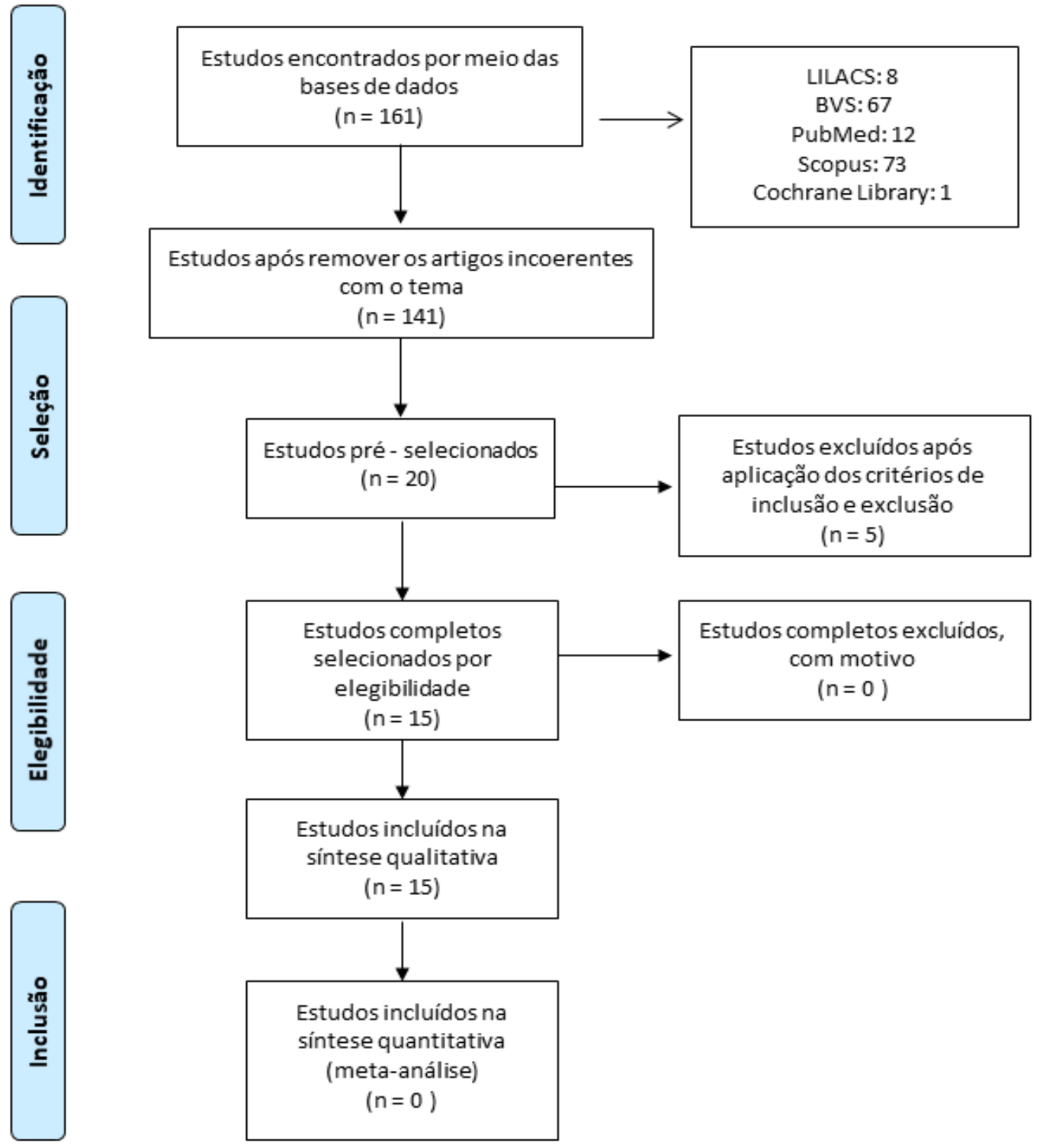

Fonte: Autores (2020) 
Os artigos selecionados para a revisão integrativa são estudos atuais sobre os atendimentos nos consultórios odontológicos e as medidas de proteção adotadas em tempos de

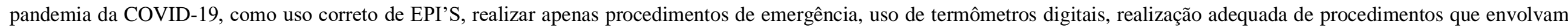
aerossol, dentre outros métodos. Os resultados foram descritos no Quadro 1 para melhor análise:

Quadro 1. Artigos selecionados de acordo com a base de dados, título, autor e conclusão.

\begin{tabular}{|c|c|c|c|c|}
\hline $\begin{array}{l}\text { Base de } \\
\text { dados }\end{array}$ & Ano & Título & Autor & Conclusão \\
\hline$B V S$ & 2020 & $\begin{array}{l}\text { Consideraciones para la Atención } \\
\text { de Urgencia Odontológica y } \\
\text { Medidas Preventivas para COVID- } \\
19 \text { (SARS-CoV 2) }\end{array}$ & Cristóbal Araya-Salas & $\begin{array}{l}\text { Em caso de emergência odontológica, se faz necessário a informação do } \\
\text { histórico médico do paciente, a fim de conhecer os possíveis sintomas do } \\
\text { coronavírus. Caso o paciente tenha algum desses sintomas no seu histórico, } \\
\text { estratégias especiais devem ser adotadas no atendimento durante a pandemia. }\end{array}$ \\
\hline$B V S$ & 2020 & $\begin{array}{l}\text { Disposable customized aerosol } \\
\text { containment chamber for oral } \\
\text { cancer biopsy: A novel technique } \\
\text { during COVID-19 pandemic. }\end{array}$ & $\begin{array}{l}\text { Sudhendu Sharma; Deepak } \\
\text { Singh; Avadhesh Yadav. } \\
\text { Jyotiranjan Swain; Sundeep } \\
\text { Kumar; Deep K. Jain; Puneet } \\
\text { Prakash. }\end{array}$ & $\begin{array}{l}\text { A câmara testada para contenção de aerossol se mostrou eficiente. Não foi } \\
\text { apresentado sufocamento ou desconforto pelo paciente. A câmera foi projetada } \\
\text { de forma a não ter contato entre o interior aerossolizado da câmera com o } \\
\text { ambiente externo durante todo o procedimento, diminuindo a probabilidade de } \\
\text { infecção pelo coronavírus. }\end{array}$ \\
\hline$B V S$ & 2020 & $\begin{array}{lcc}\text { Impacto del } & \text { COVID-19 } & \text { (SARS- } \\
\text { CoV-2) a } & \text { Nivel } & \text { Mundial, } \\
\text { Implicancias } & \text { y } & \text { Medidas } \\
\text { Preventivas en la Práctica Dental y } \\
\text { sus Consecuencias Psicológicas en } \\
\text { los Pacientes }\end{array}$ & Marcial Guiñez Coelho & $\begin{array}{l}\text { Fica claro nesse estudo que o aerossol é um grande disseminador do } \\
\text { coronavírus, podendo ficar no ar por horas. Por isso, se faz necessário o } \\
\text { cuidado com a produção desses aerossóis na prática odontológica e o uso } \\
\text { corretos dos EPI’s, como por exemplo a máscara n95. }\end{array}$ \\
\hline$B V S$ & 2020 & $\begin{array}{l}\text { COVID-19 e a Odontologia na } \\
\text { prática atual }\end{array}$ & Kátia Santos, Marcelo Barbosa & $\begin{array}{l}\text { Enxaguatórios bucais e lavagem das mãos são métodos já adotados. O uso de } \\
\text { termômetros digitais e os testes rápidos sorológicos podem ser uma prática } \\
\text { preventiva e ajudar na não disseminação do novo vírus. }\end{array}$ \\
\hline$B V S$ & 2020 & $\begin{array}{l}\text { Protocols to control contamination } \\
\text { and strategies to optimize the } \\
\text { clinical practice in Restorative } \\
\text { Dentistry during the COVID-19 } \\
\text { pandemic }\end{array}$ & $\begin{array}{l}\text { Ana Jurema, Rafael Rocha, } \\
\text { Mariane Mailart, Maurício } \\
\text { Souza, Sérgio Gonçalves, } \\
\text { Taciana Caneppele, Eduardo } \\
\text { Bresciani. }\end{array}$ & $\begin{array}{l}\text { A prática odontológica durante a pandemia do COVID-19 abriu portas para } \\
\text { atendimentos minimamente invasivos. Desde o surto do vírus da } \\
\text { imunodeficiência (HIV), muito se tem prezado para um atendimento higiênico } \\
\text { e livre de infecções cruzadas. Agora, com o coronavírus, além dos } \\
\text { procedimentos de biossegurança especialmente relacionadas ao aerossol e o }\end{array}$ \\
\hline
\end{tabular}




\begin{tabular}{|c|c|c|c|c|}
\hline & & & & $\begin{array}{l}\text { espaçamento entre os pacientes, espera-se que com o auxílio da ciência, } \\
\text { possam ser apresentadas estratégias para um atendimento cada dia mais } \\
\text { seguro. }\end{array}$ \\
\hline LILACS & 2020 & $\begin{array}{l}\text { Assessment of dental care during } \\
\text { the COVID-19 pandemic in Turkey } \\
\text { and future projections }\end{array}$ & $\begin{array}{l}\text { Mehmet Ali Kiliçarslan, Figen } \\
\text { Şenel, Mutlu Özcan }\end{array}$ & $\begin{array}{l}\text { Com a chegada da pandemia da COVID-19 mecanismos foram necessários. } \\
\text { As práticas odontológicas já são bem rigorosas, como as regras de desinfecção } \\
\text { e esterilização controladas ao mais alto nível. No entanto, as turbinas usadas } \\
\text { na maioria dos tratamentos odontológicos geram aerossóis e isso têm suscitado } \\
\text { preocupações, o aumento da quantidade de equipamentos aumentou o custo } \\
\text { dos serviços e espera-se que os protocolos sejam duradouros na odontologia } \\
\text { pós pandemia. }\end{array}$ \\
\hline LILACS & 2020 & $\begin{array}{l}\text { Knowledge, Awareness, and } \\
\text { Practice of Dentists in Preventing- } \\
\text { Novel Corona Virus (COVID-19) } \\
\text { Transmission - A Questionnaire } \\
\text { Based Cross-Sectional Survey }\end{array}$ & $\begin{array}{l}\text { Kavalipurapu } \text { Teja, Kaligotla } \\
\text { Vasundhara, } \quad \text { Gummuluri } \\
\text { Sriram }\end{array}$ & $\begin{array}{l}\text { Os dentistas têm um conhecimento adequado sobre a doença COVID-19. A } \\
\text { maioria deles faz o seu melhor para evitar a transmissão da doença ressaltando } \\
\text { a importância de seguir o protocolo oficial e realizar apenas procedimentos de } \\
\text { emergência durante a pandemia, adiando os procedimentos eletivos. }\end{array}$ \\
\hline $\begin{array}{c}P U B M E \\
D\end{array}$ & 2020 & $\begin{array}{l}\text { Biological and social aspects of } \\
\text { Coronavirus } \quad \text { Disease } 2019 \\
\text { (COVID-19) related to oral health. }\end{array}$ & 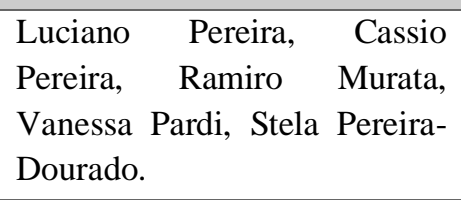 & $\begin{array}{l}\text { As características do novo coronavírus tornam o consultório odontológico } \\
\text { como alto risco para infecções. O dentista deve usar máscaras cirúrgicas e EPIs } \\
\text { clínicos completos. Procedimentos envolvendo aerossol devem ser agendados } \\
\text { para a última consulta do dia. }\end{array}$ \\
\hline $\begin{array}{l}\text { PUBME } \\
\quad \text { D }\end{array}$ & 2020 & $\begin{array}{l}\text { Covid-19: The aftermath for } \\
\text { orthodontics. }\end{array}$ & Flavia Artese & $\begin{array}{l}\text { Gotículas de saliva e aerossóis são gerados no atendimento odontológico, } \\
\text { apesar de todas as normas de proteção padrão, elas não são suficientes para } \\
\text { prevenir o contágio pela COVID-19. Profissionais de destaque têm proposto } \\
\text { um aumento no uso da ortodontia digital e do acompanhamento de pacientes } \\
\text { por meio da teleodontologia. As máquinas ou a inteligência artificial são } \\
\text { mecanismos de ajuda e não de substituição da humanidade. Em consequência } \\
\text { da pandemia, a ortodontia irá recuperar seu papel como uma verdadeira } \\
\text { especialidade. }\end{array}$ \\
\hline $\begin{array}{c}P U B M E \\
D\end{array}$ & 2020 & $\begin{array}{l}\text { Teledentistry support in COVID-19 } \\
\text { oral care }\end{array}$ & $\begin{array}{l}\text { Gabriel Telles-Araujo, Raquel } \\
\text { Caminha, Monira Kallás, } \\
\text { Paulo Santos. }\end{array}$ & $\begin{array}{l}\text { A telemedicina não é um substituto para a consulta presencial, e tem como } \\
\text { objetivo principal apoiar o Sistema Único de Saúde (SUS) durante a pandemia } \\
\text { da COVID-19. Sempre que uma consulta física for necessária, as normas de } \\
\text { saúde do órgão regulador devem ser seguidas. }\end{array}$ \\
\hline SCOPUS & 2020 & $\begin{array}{l}\text { Coronavirus disease } 2019 \text { and } \\
\text { dental care for older adults: New } \\
\text { barriers require unique solutions }\end{array}$ & $\begin{array}{l}\text { Leonardo Marchini, Ronald } \\
\text { Ettinger. }\end{array}$ & $\begin{array}{l}\text { A teleodontologia pode ser importante para avaliar alguns pacientes. Também } \\
\text { precisamos educar nossos idosos e seus responsáveis sobre as estratégias que } \\
\text { estão sendo usadas para minimizar o risco do paciente de desenvolver infecção }\end{array}$ \\
\hline
\end{tabular}




\begin{tabular}{|c|c|c|c|c|}
\hline & & & & $\begin{array}{l}\text { por COVID-19. As estratégias incluem avaliação dos sintomas, a temperatura, } \\
\text { o distanciamento social e o uso de EPI. }\end{array}$ \\
\hline SCOPUS & 2020 & $\begin{array}{l}\text { Protective device to reduce aerosol } \\
\text { dispersion in dental clinics during } \\
\text { the COVID-19 pandemic }\end{array}$ & $\begin{array}{l}\text { R. Teichert-Filho, C. } \\
\text { Baldasso, M. } \\
\text { Gomes. }\end{array}$ & $\begin{array}{l}\text { O dispositivo que consiste em uma estrutura acrílica translúcida rígida } \\
\text { (polimetaclato de metila), envolvendo cabeça, pescoço e tórax do paciente é } \\
\text { um recurso complementar de baixo custo para uso em conjunto com EPI } \\
\text { padrão, para reduzir a transmissão de SARS-CoV-2 no ambiente odontológico. }\end{array}$ \\
\hline SCOPUS & 2020 & $\begin{array}{l}\text { Oral radiology practice in dental } \\
\text { schools during the COVID-19 } \\
\text { pandemic: What will be the new } \\
\text { normal? }\end{array}$ & $\begin{array}{l}\text { Rocharles Fontenele, Amanda } \\
\text { Gomes, Deborah Freitas. }\end{array}$ & $\begin{array}{l}\text { Durante e após a pandemia da COVID-19 a prática da clínica nas } \\
\text { universidades será um desafio. A recomendação é de que as mudanças } \\
\text { atualmente adotadas para a proteção individual dos profissionais e dos } \\
\text { pacientes devem ser incorporadas à prática clínica a fim de reduzir o risco de } \\
\text { infecções cruzadas. }\end{array}$ \\
\hline SCOPUS & 2020 & $\begin{array}{l}\text { Assessment of the Economic and } \\
\text { Health-Care Impact of COVID-19 } \\
\text { (SARS-CoV-2) on Public and } \\
\text { Private Dental Surgeries in Spain: } \\
\text { A Pilot Study }\end{array}$ & $\begin{array}{l}\text { Cintia Chamorro-Petronacci, } \\
\text { Carmen } \quad \text { Carreras-Presas, } \\
\text { Adrianar Sanz-Marchena, } \\
\text { María Rodríguez-Fernández, } \\
\text { José Suárez-Quintanilla, Berta } \\
\text { Rivas-Mundiña, Juan Suárez- } \\
\text { Quintanilla e Mario Pérez- } \\
\text { Sayáns. }\end{array}$ & $\begin{array}{l}\text { A pandemia da COVID-19 apresentou repercussões econômicas para a } \\
\text { odontologia já que o único tratamento disponível durante o estado de alarme } \\
\text { era o de urgência. O número de pacientes tratados também diminuiu e a } \\
\text { introdução de novos EPI's como o termômetro digital e o aumento na } \\
\text { quantidade dos equipamentos para segurança individual anteriormente } \\
\text { utilizados provocaram o aumento de custos. Com isso, os resultados } \\
\text { apresentados indicam a importância dos atendimentos odontológicos e a } \\
\text { necessidade da devida ajuda econômica. }\end{array}$ \\
\hline $\begin{array}{c}\text { COCHR } \\
\text { ANE }\end{array}$ & 2020 & $\begin{array}{l}\text { Interventions to reduce } \\
\text { contaminated aerosols produced } \\
\text { during dental procedures for } \\
\text { preventing infectious diseases. }\end{array}$ & $\begin{array}{l}\text { Sumanth Nagraj, Prashanti } \\
\text { Eachempati, Martha Paisi, } \\
\text { Mona Nasser, Gowri } \\
\text { Sivaramakrishnan, Jos H } \\
\text { Verbeek. }\end{array}$ & $\begin{array}{l}\text { A transmissão da COVID-19 na clínica odontológica ocorre principalmente } \\
\text { por contato direto com as gotículas respiratórias e contato indireto com } \\
\text { superfícies. Para evitar a contaminação se faz necessário o uso completo de } \\
\text { EPI's (luvas, óculos de proteção, jaleco) e para evitar contaminação por } \\
\text { aerossol, a utilização de agentes antimicrobianos. }\end{array}$ \\
\hline
\end{tabular}

Fonte: Autores. 


\section{Discussão}

A literatura mostra um compilado de métodos adotados nos atendimentos odontológicos durante a pandemia da COVID19 e as ações de biossegurança que melhor se adequam a esse momento. Os autores Santos \& Barbosa, 2020 concordam que, frente aos desafios do tempo atual, os profissionais de odontologia tiveram que restringir seus atendimentos para apenas emergências e urgências. Como consequência, Chamorro-Petronacci et al. (2020) deixa explícitas as repercussões econômicas na odontologia que a pandemia do coronavírus (SARS-COV-2) trouxe.

Apesar disso, benefícios para um atendimento seguro são encontrados utilizando algumas estratégias específicas. Teichert-Filho et al. (2020) fala sobre a importância do equipamento de proteção individual (EPI) completo, com óculos de proteção, face shield, touca/gorro, jaleco/avental impermeável, pijamas cirúrgicos, máscaras e pro-pé. Atrelado a isso, uma estrutura acrílica translúcida rígida, na cadeira odontológica, que envolva cabeça, pescoço e tórax do paciente, é um dispositivo complementar que ainda deve ter sua eficácia comprovada.

É relevante ressaltar a importância, baseando-se em Araya-Salas (2020), da adoção de procedimentos que promovam mais segurança no atendimento. A exemplo dos exames de imagem, o autor recomenda o uso preferencial das radiografias panorâmicas, durante os atendimentos na pandemia, a fim de diminuir os riscos de contaminação, já que são imagens extra orais. Por outro lado, Fontenele, Gomes e Freitas (2020) sugerem que, na aquisição de radiografias intraorais obrigatórias, deve-se pedir ao paciente que enxágue a boca com agentes oxidantes antes da imagem ser adquirida.

A autora Jurema et al. (2020) afirma que a melhor forma de prevenção da COVID-19 é o isolamento social, concorda sobre a importância do uso de equipamentos de proteção individual (EPI) e desinfecção das mãos com álcool gel 70\%, cita também o isolamento absoluto de borracha como as melhores ações adotadas durante os atendimentos. Ainda, visando a proteção do paciente e do profissional, os autores Sharma et al. (2020), a partir de um ensaio clínico, constataram a relevância do uso de uma câmera de contenção de aerossol descartável personalizada, limitando a contaminação com o aerossol produzido pela caneta de alta rotação.

Para Santos e Barbosa, 2020, a adoção das precauções supracitadas impactaria na prevenção e diminuição de números de pessoas contaminadas não só pelo recente coronavírus, mas por outras doenças infectocontagiosas, dentro do consultório odontológico, promovendo a saúde geral do paciente. Além disso, Santos e Barbosa, 2020 indicam o uso de testes rápidos pelos cirurgiões-dentistas, isso impactaria no controle melhor do vírus e aumentaria o índice de diagnósticos precoces, diminuindo a quantidade de pessoas infectadas e a contaminação cruzada dentro do consultório, de uma forma rápida e não invasiva. Com relação a utilização de termômetros, o autor acredita que também contribui na prática clínica referente aos tratamentos dentários e ao bem-estar total do paciente. Portanto, o cirurgião-dentista tem capacidade para agir na prevenção e ajudar os diversos profissionais de saúde no combate à pandemia.

Apesar de avanços na construção de protocolos de atendimento que impactem de forma positiva, Kiliçarslan et al. (2020) acredita que a profissão ainda se depara com algumas limitações. Os autores Guiñez-Coelho et al. (2020) e Nagraj et al. (2020) acrescentam que não existem estudos suficientes de substâncias, um diagnóstico eficientemente rápido e protocolos específicos para o tratamento e prevenção da COVID-19. Por isso, estão sendo adaptados mecanismos já existentes, decorrentes de anos de estudos sobre biossegurança, sendo assim, cada país elaborou os seus protocolos para manter os tratamentos odontológicos de acordo com as suas realidades e políticas de saúde.

No Brasil, o Conselho Federal de Odontologia (CFO) (2020) em seu manual de boas práticas em biossegurança para ambientes odontológicos, realiza recomendações para cirurgiões dentistas no âmbito da clínica odontológica. Dentre elas, algumas precauções padrões, como dispenser de álcool em gel nos ambientes da clínica prezando pelo fácil acesso ao paciente, pia e sabonete, na recepção, para higienização das mãos e rosto. Promover o distanciamento, espaçando cadeiras 1 metro por pessoa na sala de espera. Além disso, medidas para diminuição da produção de aerossol também foram citadas, como realizar a 
sucção constante de saliva, com bomba à vácuo, evitar a utilização da seringa tríplice e optar por secar com algodão ou gaze.

No que diz respeito a limpeza de áreas comuns, o manual do CFO recomenda realizar a desinfecção das superfícies do consultório e ambientes utilizados pelo paciente, antes das atividades clínicas e no intervalo entre um paciente e outro, utilizando como agentes de desinfecção hipoclorito de sódio a 1\%, quaternário de amônio e biguanida, glucoprotamina e álcool $70 \%$. Ademais, se faz o alerta para a ordem correta de remoção dos EPI's para evitar a contaminação, que deve ser: remover as luvas, em seguida a proteção facial, depois retirar o jaleco ou avental e finalizar pela remoção do gorro e máscara, higienizando as mãos e o rosto ao final.

Em relação ao atendimento feito por estudantes, os autores Kiliçarslan et al. (2020) e Telles-Araujo et al. (2020) acreditam que vai ser uma situação delicada, com algumas limitações. As práticas devem ser remodeladas e o treinamento teórico deve aproveitar as oportunidades que a tecnologia oferece ao máximo. Ainda referente à tecnologia, a Resolução 226/2020 do Conselho Federal de Odontologia (2020) reconhece a teleorientação como uma ferramenta que permite os dentistas a fazerem uma triagem a distância com orientação, monitorização e encaminhamento.

Em protocolo oficial, a Associação Brasileira de Ensino Odontológico (ABENO) (2020) sugere cuidados no ambiente acadêmico para garantir um aprendizado seguro para docentes e discentes. Entre alguns métodos, destaca-se no espaço clínico, novos protocolos para o uso de ar-condicionado, estratégias para o controle da produção de aerossol e área específica para paramentação e desparamentação. No que diz respeito a rotina universitária, sugere-se que a disposição das cadeiras na sala de aula deve estar sinalizada por fita no chão e no laboratório, bancadas de trabalho devem ser intercaladas para respeitar distância mínima de 2 m entre os ocupantes

No entanto, Telles-Araujo et al. (2020) também deixa claro que é necessário esclarecer que a telemedicina, assim como a teleodontologia, é um paliativo e serve apenas para apoiar o Sistema Único de Saúde (SUS) durante a pandemia. A consulta física e presencial é extremamente importante e necessária, não podendo ser substituída, e deve ser regulamentada pelos órgãos de saúde. Além disso, Marchini \& Ettinger (2020) afirmam que é importante o dentista informar o paciente sobre a limitação da consulta e também a registrar detalhadamente.

Por se tratar de um momento crítico atual, em que os cientistas ainda estão desvendando esse novo vírus e em processo de formação de conhecimento, algumas dificuldades foram encontradas no desenvolvimento desse trabalho, como técnicas sem comprovação de eficácia garantida.

Portanto, o estudo e análise das novas estratégias de biossegurança durante e após o coronavírus, para o atendimento odontológico, são de extrema relevância para a comunidade acadêmica e para os cirurgiões-dentistas nas suas clínicas. Os conhecimentos adquiridos, utilizados na prática odontológica, podem interferir de forma positiva nos atendimentos odontológicos a curto e longo prazo.

\section{Conclusão}

Pode-se concluir que a literatura concorda que a melhor forma de prevenção é o isolamento social, atrelado a diversos métodos que podem ser adotados nos atendimentos odontológicos durante a pandemia da COVID-19. Portanto, em todos os atendimentos odontológicos, os protocolos de biossegurança devem ser seguidos rigorosamente pelo cirurgião-dentista e sua equipe, garantindo um ambiente adequado e seguro a todos os seus pacientes.

Além disso, fica evidente que, nesse momento de descobertas sobre o coronavírus, os cirurgiões-dentistas podem contribuir de forma significativa com a comunidade científica e com a população, visto que são profissionais que atuam rotineiramente em ambulatórios públicos ou privados, sempre com indicação de uso de paramentação biossegurança, em decorrência da alta exposição a doenças e infecções antes mesmo da COVID-19. Ademais a busca constante por informações relevantes e análise dos novos métodos é indispensável. 


\section{Referências}

Araya-Salas, C. (2020). Consideraciones para la atención de urgencia odontológica y medidas preventivas para COVID-19 (SARS-CoV 2). International journal of odontostomatology 14(3), 268-270. http://dx.doi.org/10.4067/S0718-381X2020000300268.

Artese, F. (2020). Covid-19: The aftermath for orthodontics. Dental Press Journal of Orthodontics, 25(2), 7-8. https://dx.doi.org/10.1590/2177-6709.25.2.007008.edt.

Associação Brasileira de Ensino Odontológico. (2020). Consenso ABENO: Biossegurança no ensino odontológico pós-pandemia da COVID19. ABENO. http://www.abeno.org.br/arquivos/downloads/retomada_de_praticas_seguras_no_ensino_odontologico.pdf.

CFO - Conselho Federal de Odontologia. (2020). Resolução CFO 226, de 04 de junho de 2020. Dispõe sobre o exercício da Odontologia a distância, mediado por tecnologias, e dá outras providências. http://sistemas.cfo.org.br/visualizar/atos/RESOLU\%c3\%87\%c3\%83O/SEC/2020/226.

Conselho Federal de Odontologia (2020). Manual de boas práticas em biossegurança para ambientes odontológicos. CFO: Brasília. cfo-lança-Manual-de-BoasPráticas-em-Biossegurança-para-Ambientes-Odontologicos.pdf

Chamorro-Petronacci, C., Martin Carreras-Presas, C., Sanz-Marchena, A., A Rodríguez-Fernández, M., María Suárez-Quintanilla, J., Rivas-Mundiña, B. \& Pérez-Sayáns, M. (2020). Assessment of the economic and health-care impact of COVID-19 (SARS-CoV-2) on public and private dental surgeries in Spain: A pilot study. International Journal of Environmental Research and Public Health, 17(14), 5139. https://doi.org/10.3390/ijerph17145139.

Fontenele, R. C., Gomes, A. F., \& Freitas, D. Q. (2020). Oral radiology practice in dental schools during the COVID-19 pandemic: What will be the new normal?. Imaging Science in Dentistry, 50(3), 265-267. https://doi.org/10.5624/isd.2020.50.3.265.

Guiñez-Coelho, M. (2020). Impacto del COVID-19 (SARS-CoV-2) a nivel mundial, implicancias y medidas preventivas en la práctica dental y sus consecuencias psicológicas en los pacientes. International journal of odontostomatology, 14(3), 271-278. http://dx.doi.org/10.4067/S0718-381X2020000300271.

Jurema, A. L. B., Rocha, R. S., Mailart, M. C., de Souza, M. Y., de Paiva Gonçalves, S. E., Caneppele, T. M. F., \& Bresciani, E. (2020). Protocols to control contamination and strategies to optimize the clinical practice in Restorative Dentistry during the COVID-19 pandemic. Brazilian Dental Science, 23(2), 10-p. https://doi.org/10.14295/bds.2020.v23i2.2256.

Kılıçarslan, M. A., Şenel, F. Ç., \& Özcan, M. (2020). Assessment of dental care during the covid-19 pandemic in Turkey and future projections. Brazilian Dental Science, 23(2), 7. https://doi.org/10.14295/bds.2020.v23i2.2260.

Marchini, L., \& Ettinger, R.L. (2020). Coronavirus disease 2019 and dental care for older adults: New barriers require unique solutions. Journal of the American Dental Association, 151 (12) - 881-884. https://doi.org/10.1016/j.adaj.2020.08.004.

Nagraj, S. K., Eachempati, P., Paisi, M., Nasser, M., Sivaramakrishnan, G., \& Verbeek, J. H. (2020). Interventions to reduce contaminated aerosols produced during dental procedures for preventing infectious diseases. Cochrane Database of Systematic Reviews, (10). https://doi.org/10.1002/14651858.CD013686.pub2.

Pereira, L. J., Pereira, C. V., Murata, R. M., Pardi, V., \& Pereira-Dourado, S. M. (2020). Biological and social aspects of Coronavirus Disease 2019 (COVID19) related to oral health. Brazilian Oral Research, 34. https://doi.org/10.1590/1807-3107bor-2020.vol34.0041.

Santos, K. F. \& Barbosa, M. (2020). COVID-19 and Dentistry in current practice. https://doi.org/10.1590/SciELOPreprints.746.

Sharma, S. S., Singh, D. K., Yadav, A. K., Swain, J., Kumar, S., Jain, D. K., \& Prakash, P. (2020). Disposable customized aerosol containment chamber for oral cancer biopsy: A novel technique during COVID-19 pandemic. Journal of Surgical Oncology. https://doi.org/10.1002/jso.25962.

Teichert-Filho, R., Baldasso, C. N., Campos, M. M., \& Gomes, M. S. (2020). Protective device to reduce aerosol dispersion in dental clinics during the COVID19 pandemic. International endodontic journal, 53(11), 1588-1597. https://doi.org/10.1111/iej.13373.

Teja, K. V. \& Vasundhara, K. V. A. (2020). Knowledge, Awareness, and Practice of Dentists in Preventing-Novel Corona Virus (COVID-19) Transmission-A Questionnaire Based Cross-Sectional Survey. Brazilian Dental Science 23, (2). https://doi.org/10.14295/bds.2020.v23i2.2287.

Telles-Araujo, G. D. T., Caminha, R. D., Kallás, M. S., \& Santos, P. S. D. S. (2020). Teledentistry support in COVID-19 oral care. Clinics, 75. https://doi.org/10.6061/clinics/2020/e2030.

Whittemore, R., \& Knafl, K. (2005). The integrative review: updated methodology. Journal of advanced nursing, 52(5), 546-553. http://dx.doi.org/10.1111/j.1365- 2648.2005.03621.x. 